\title{
INTERVENÇÃO NUTRICIONAL PADRONIZADA EM PACIENTES HIPOGLICÊMICOS HOSPITALIZADOS
}

\author{
Silmara Rodrigues M ACH AD Oa, A na Lúcia Chal houb Chediác ROD RI GU E S , Sandra Cristine da SILVAc \\ Rafael de Oliveira ALVIM d Paulo Caleb Júnior Lima SANT OS
}

\section{RESUMO}

A ingestão de carboidratos de rápida absorção (CRA) pode ser útil para o aumento sérico de glicose. N este contexto, os principais objetivos foram avaliar a eficácia e a aplicabilidade da intervenção nutricional em situações hipoglicêmicas apresentadas por pacientes conscientes, com dieta via oral e internados em hospital geral. Setenta e seis pacientes foram el egíveis e a hipoglicemia foi definida como nível de glicemia capilar $\geq 50$ até $\leq 70 \mathrm{mg} / \mathrm{dL}$. A inter ven ção nutricional constituiu na oferta de 15 a 24 gramas de CRA. H ouve a conferência da glicemia capilar após 15-20 minutos da intervenção. A taxa de efetividade da intervenção nutricional foi de 97,6\%, durante 0 período de estudo. Conclui-se que a administração de CRA, um método não invasivo, foi aplicável em unidades de um hospital geral e foi potencialmente eficaz na restauração da glicemia capilar em pacientes hipoglicêmicos com dieta via oral e conscientes.

Descritores: H ipoglicemia. Pacientes internados. G licemia.

\section{RESUMEN}

L a ingesta de hidratos de carbono de absorción rápida (H AR ) puede ser útil a la gl ucosa sérica. E n estecontexto, los principales objetivos fueron evaluar la eficacia y aplicabilidad de la atención nutricional en situaciones de hipoglucemia en el hospital general. Setenta y seis pacientes fueron elegibles. $L$ a hipoglucemia se definió como el nivel de glucosa en sangre $\geq 50 a \leq 70$ $\mathrm{mg} / \mathrm{dL}$. Intervención nutricional que se ofrecía 15 a 24 gramos de H AR. H ubo una conferencia de glucosa en la sangre después de 15-20 minutos de acción. $L$ a tasa de eficacia de la intervención nutricional fue del 97,6\% durante el período de estudio. Seconcluye que la administración de H AR, un método no invasivo, es aplicableen las unidades dehospital general y fue potencialmente eficaz en la restauración de la glucosa en sangre en pacientes con hipoglucemia, consciente, y con dieta oral.

Descriptores $\mathrm{H}$ ipoglucemia. Pacientes internos. G lucemia.

Título: Intervención nutricional estandarizado en pacientes con hipoglucemia y hospitalizados.

\section{ABSTRACT}

The ingestion of rapid absorption car bohydrates (RAC) can be useful to increase ser um gl ucose. This sudy aimed to assess the effectiveness and applicability of nutritional intervention in hypoglycemic situations suffered by conscious inpatients in a general hospital who were receiving oral diet. Seventy-six patients were eligible $\mathrm{H}$ ypoglycemia was defined as a capillary glucoselevel of $\geq 50$ to $\leq 70 \mathrm{mg} / \mathrm{dL}$. N utritional intervention consisted in providing 15-24 g of RAC. Thelevel of capillary glucose was checked after 15 to 20 minutes of the intervention. During the studied period, the rate of effectiveness of the nutritional inter vention was $97.6 \%$. R esults show that the administration of RAC, a non-invasivemethod, was applicable in a general hospital and was also potentially effective in restoring capillary glucose levels in conscious hypoglycemic inpatients receiving an oral diet.

Descriptors: $\mathrm{H}$ ypogl ycemia. Inpatients. B lood glucose

Title: Standardiz ed nutritional intervention in hypoglycemic inpatients.

\footnotetext{
a M BA em Alimentação, M estranda pelo Programa de Pós-G raduação em Ciências da Saúde da U niver sidade Federal de São Paulo, Nutricionista Clínica da U nidade de Terapia Intensiva da Sociedade Beneficente de Senhoras do H ospital Sírio Libanês (SBS-HSL), São Paulo, Brasil.

b Especial ista em A dministração H ospitalar e em N utrição Cardiológica, Coordenadora do Serviço de A limentação da SBS-H SL, São Paulo, Brasil.

' D outora em Enfermagem, Enfermeira - G erente de Qualidade da SBS-HSL, São Paulo, Brasil.

d Especialista em Reabilitação Cardiovascular, Educador Físico - Instituto do Coração (InCor) do H ospital das Clínicas da Faculdade de M edicina da Universidade de São Paulo (H C-F M U SP), São Paulo, Brasil.

e D outor em Ciências, Farmacêutico Bioquímico - InCor do HC-F M USP, São Paulo, Brasil.
} 


\section{INT RODUÇÃO}

A hipoglicemia é um fator limitante para o controle glicêmico no diabetes ${ }^{(1,2)}$, causando morbidade na maioria das pessoas com diabetes mellitus (DM) tipo 1 e muitas vezes é fatal no DM tipo 2. $M$ anter a glicemia nos limites de normalidade diminui os riscos de ocorrência das complicações mais frequentes dos DM tipos 1 e 2, tais como: retinopatia, nefropatia, neuropatia e também pode reduzir eventos cardiovasculares significativamente ${ }^{(3)}$.

A glicose é o principal combustível metabólico para o cérebro e, como este não pode sintetizála ou armazenar glicogênio para a sua produção, cria-se uma dependência contínua da glicose disponível na circulação sanguínea ${ }^{(4)}$. Q uando os seus níveis encontram-se diminuídos na circulação, as atividades cerebrais tornam-se limitadas, há um comprometimento do metabolismo cerebral e até mesmo da sobrevivência. Os efeitos deste estado são deletérios e o controleglicêmico bastante complexo(1).

Os sinais e sintomas da hipoglicemia são, na maioria, neurológicos decorrentes da privação de glicose à atividade cer ebral e, incluem sensações de fraqueza, fadiga, dificuldade para pensar, confusão, mudanças comportamentais e labilidade emocional. Se esta situação hipoglicêmica for prolongada, podem ocorrer convulsões, perda da consciência, lesão cer ebral e até mesmo a morte ${ }^{(1,4)}$.

A hipoglicemia iatrogênica resulta da ação absoluta ou relativa de insulina em excesso e, deste modo, compromete a regulação do metabolismo da glicose nos D M tipos 1 e $2^{(1,5)}$. Os fatores de risco relevantes que comprometem a contra regulação de glicose são bem estabelecidos no D M e incluem: 1) deficiência insulínica; 2) história de hipoglicemia severa, hipoglicemia desconhecida ou ambos; e 3) ter apia glicêmica ag ressiva, como evidenciada por baixas concentrações de hemoglobina glicada, metas glicêmicas baixas ou ambos ${ }^{(6)}$.

Ressalta-se que a hipoglicemia está primeiramente relacionada com a administração de insulina. Porém, mudanças no consumo al imentar, atividade física e medicamentos também podem contribuir para esta situação(7). E mbora rígidas definições de hipoglicemia sejam úteis em estudos epidemiológicos, definições mais flexíveis são suficientes no tratamento do diabetes ${ }^{(8)}$.

U $m$ dos tratamentos da hipoglicemia é a ingestão de glicose ou de alimento contendo car- boidrato de rápida absorção. Ressalta-se que 0 estado do carboidrato - sólido ou líquido - utilizado para o tratamento não interfere na eficácia ${ }^{(9)}$. De acordo com a A ssociação A mericana do D iabetes (ADA ), em situações de hipoglicemia, devem ser ofertados alimentos que contenham de 15 a 20 gramas de carboidrato de rápida absorção, tais como: suco de laranja, suco de uva, glicose gel, torta doce, balas de goma, tabletes de mel ou refrigerante(10).

A ingestão de carboidratos de rápida absorção pode ser facilmente aplicável para o aumento sérico de glicose, porém há escassez de estudos na literatura que avaliem esta intervenção nutricional. N este contexto, os principais objetivos foram quantificar a eficácia e a aplicabilidade da intervenção nutricional em situações hipoglicêmicas apresentadas por pacientes internados em um hospital geral.

\section{MÉTODOS}

0 estudo foi realizado em todas as unidades de internação, críticas e semi-intensivas de um hospital de grande porte, localizado em São Paulo, Brasil. Foi desenvolvido pelos nutricionistas clínicos durante cinco meses (outubro de 2007 a fevereiro de 2008), após orientação sobre a aplicação da intervenção nutricional na hipoglicemia. Tratou-se de uma amostragem de conveniência a qual consiste em recrutar pacientes que preencham os critérios de seleção dentro de um intervalo de tempo e não é uma técnica de amostragem probabilística. Este estudo foi aprovado pelo Comitê de Ética em Pesquisa da Sociedade Beneficente de Senhoras Hospital Sírio Libanês de São Paulo (CE Pesq-HSL 35).

Setenta e seis pacientes foram el egíveis para este estudo. Foram incluídos os pacientes que apresentaram hipoglicemia (glicemia capilar $\geq 50$ até $\leq 70 \mathrm{mg} / \mathrm{dL}$ ), que estavam conscientes e com dieta via oral. Não houve restrição quanto ao gênero, à idade, à causa da hipoglicemia ou ao motivo da internação. Foram excluídos os pacientes inconscientes, sem dieta via oral ou com oferta de outros alimentos não inclusos no protocolo segundo a ADA.

Em relação à intervenção nutricional clíni$c a$, foram ofer ecidos alimentos contendo carboidratos $(\mathrm{CHO})$ de rápida absorção preconizados pela ADA: $200 \mathrm{~mL}$ de refrigerante a base de cola $(21 \mathrm{~g}$ de $\mathrm{CHO}$ ), ou $200 \mathrm{~mL}$ de refrigerante de guaraná 
( $20 \mathrm{~g}$ de $\mathrm{CHO}$ ), ou $30 \mathrm{~g}$ de mel ( $24 \mathrm{~g}$ de $\mathrm{CHO}$ ), ou $200 \mathrm{~mL}$ de suco de maçã industrializado (19 g de $\mathrm{CHO}$ ), ou $150 \mathrm{~mL}$ de suco delaranja ( $15 \mathrm{~g}$ de $\mathrm{CHO}$ ) ou purê de fruta de maçã ( $17 \mathrm{~g}$ de $\mathrm{CHO}$ ). Estes foram selecionados de acordo com a consistência da dieta prescrita, por exemplo: purê de fruta de maçã para pacientes com prescrição médica de dieta pastosa. Para conhecimento da intervenção nutricional, houve divulgação institucional em rede, treinamento da equipe de copa e de enfermagem, criação de formulários com o fluxograma e distribuição destes nas unidades de inter nação.

0 tempo sugerido pela ADA para observar 0 aumento da resposta glicêmica após a administração do alimento ao paciente é de 15 a 20 minutos. N este estudo foi realizada medida de glicemia capilar (dextro) nos primeiros 15-20 minutos, porém medições de até 40 minutos foram aceitas. Valores de glicemia capilar $>70 \mathrm{mg} / \mathrm{dL}$ foram considerados como resposta glicêmica adequada à intervenção nutricional.

A equipe de enfermagem atendeu imediatamente os pacientes em quadro de hipoglicemia e, quando a intervenção nutricional padronizada foi aplicada, 0 alimento escol hido foi ingerido em sua totalidade. Observado o desaparecimento dos sinais e sintomas hipoglicêmicos com a administração do alimento por via oral e checada a dextro, a equipe de enfer magem relatava ao médico responsável pelo paciente. Em situações nas quais não houve melhora dos sinais e sintomas clínicos, o médico responsável foi imediatamente comunicado, a fim de sinalizar outra conduta.

Os nutricionistas clínicos identificaram os pacientes com quadro de hipoglicemia pelo acompanhamento diário via prontuário. A intervenção nutricional realizada pela equipe de enfermagem, assim como a identificação do paciente com hipoglicemia pelos nutricionistas, ocorriam durante 24 horas por dia e 7 dias na semana. Posterior mente, reportaram em formulário específico: o número do leito, o valor de dextro antes, 0 al imento oferecido ao paciente que apresentou hipoglicemia e 0 val or de dextro após a aplicação da intervenção.

A taxa de aplicabilidade da intervenção nutricional foi avaliada através da relação: número de pacientes elegíveis e atendidos pela intervenção nutricional / número total de pacientes elegíveis (atendidos e não pela intervenção nutricional). A taxa de eficácia foi avaliada pela relação: número de pacientes com glicemia capilar dentro do limite de normalidade após aplicação da inter venção nutricional / número total de pacientes submetidos à intervenção nutricional.

\section{RESULTADOS}

D os 76 pacientes selecionados (idade média de 64,3 $\pm 12,1$ anos), havia 47 homens e 29 mulheres. D urante os cinco meses de estudo, 140 pacientes apresentaram hipoglicemia e 76 foram submetidos à intervenção nutricional.

No primeiro mês de estudo, a taxa de aplicabilidade foi de $35,0 \%$ (14/40). N os quatro meses posteriores foram de: $50,0 \%$ (13/26), 73,9\% (17/ 23), $51,7 \%$ (15/29) e $77,3 \%$ (17/ 22) (Figura 1). Ao final do período de estudo, a média da aplicabilidade mensal foi de $57,6 \%$ (F igura 1). Os principais motivos para a não adesão da intervenção nutricional foram a ingestão de alimentos não recomendados pelo protocolo ou a conduta médica de utilizar infusão de soro glicosado.

A s taxas de eficácia foram de $100,0 \%$ em quatro meses e de $88,2 \%$ (15/17) em um dos meses (terceiro mês) da intervenção nutricional. Durante o período de estudo, a média da taxa de eficácia foi de $97,6 \%$ (Figura 1). Todos os pacientes que receberam 0 tratamento dietoterápico apresentaram um dextro dentro do limite de normalidade, exceto dois pacientes no terceiro mês. No entanto, não houve associação deste fato com qualquer outra evidência clínica destes pacientes.

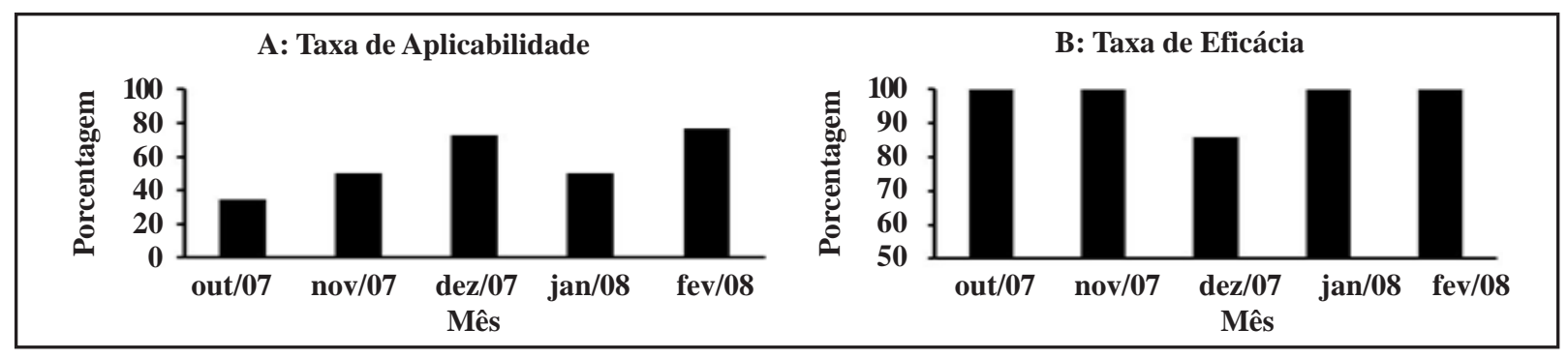

Figura 1 - T axa de aplicabilidade (A) e taxa de eficácia (B) da inter venção nutricional em um hospital de São Paulo no período de outubro de 2007 a fevereiro de 2008. 
Durante os cinco meses, 140 pacientes apresentaram hipoglicemia e 76 foram submetidos à intervenção nutricional. As taxas de aplicabilidade foram de $35,0 \%$ (14/40), 50,0\% (13/26), 73,9\% (17/ 23), 51,7\% (15/29) e 77,3\% (17/ 22), respectivamente. As taxas de eficácia foram de 100,0\% em quatro meses e de $88,2 \%$ (15/17) em um dos meses (terceiro mês).

\section{DISCUSSÃO}

Os principais resultados observados foram que a ingestão de carboidratos de rápida absorção é aplicável em um hospital geral de grande porte e esta é eficaz em situações hipoglicêmicas apresentadas por pacientes orientados, conscientes e com dieta via oral.

Durante o primeiro mês do estudo, a taxa de aplicabilidade de $35,0 \%$ se deve a ausência de conhecimento da equipe multiprofissional sobre a intervenção nutricional em intercorrências hipoglicêmicas. A tendência foi aumentar a aplicação deste plano após o treinamento e divulgação institucional. N os quatro meses seguintes do estudo, o aumento da aplicação do plano foi observado e a média da aplicabilidade foi de $57,6 \%$. Esta média ainda aumentou pós-estudo, com o permanente treinamento e a divulgação para a equipe multiprofissional.

U m estudo comparando a administração via oral de 20 gramas de glicose e 20 gramas de carboidrato presente no leite e no suco de laranja concluiu que a frutose presente no suco e a galactose do leite são tão efetivas quanto à glicose para promover o aumento adequado da glicose sanguínea(1). Outro estudo relatou que 10 gramas de glicose via oral aumenta o nível da glicose sanguínea de $60 \mathrm{mg} / \mathrm{dL}$ para $97 \mathrm{mg} / \mathrm{dL}$, e 20 gramas de glicose aumenta de $58 \mathrm{mg} / \mathrm{dL}$ para $122 \mathrm{mg} / \mathrm{dL}$. As duas abordagens são eficazes no tratamento de indivíduos adultos em hipoglicemia, porém, há necessidade de aval iação posterior a oferta(11). A associação de proteína e carboidrato para o tratamento de hipoglicemia foi avaliada e indicou não acelerar a resposta glicêmica ${ }^{(12,13)}$.

E $m$ relação à taxa de eficácia, todos os pacientes que receberam o tratamento dietoterápico apresentaram um dextro dentro do limite de normalidade, exceto dois pacientes no terceiro mês. Porém, não se correlacionou 0 achado com qualquer evidência clínica destes pacientes. Sabe-se ain- da que as causas hipoglicêmicas podem interagir com a resposta glicêmica à intervenção nutricional aplicada ${ }^{(14)}$. Este item não foi avaliado pelo estudo, assim como a estratificação por faixas etárias, gênero, etnia, susceptibilidade genética e alimento utilizado. D este modo, estes são alguns limitantes do presente estudo, que possivelmente poderiam evidenciar importantes covariáveis da resposta glicêmica(15-20). Em contrapartida, a média da taxa de eficácia, de $88,2 \%$, pode indicar 0 potencial da utilização de carboidrato de rápida absorção advindo de diversos alimentos, em pacientes de ambos gêneros, de idades variadas e de diversas causas hipoglicêmicas.

\section{CONCLUSÕES}

N os últimos anos, o suporte nutricional evoluiu extraordinariamente e consequentemente contribui para melhores tratamentos dos pacientes, principalmente na área de cuidados intensivos. N este estudo, a simples intervenção nutricional foi capaz de prevenir os possíveis efeitos deletérios da hipoglicemia. N este cenário, a administração de carboidratos de rápida absorção, um método não invasivo, foi aplicável em unidades de um hospital geral e foi potencialmente eficaz na restauração da glicemia capilar em pacientes hipoglicêmicos com dieta via oral e conscientes.

\section{REFERÊNCIAS}

1 Cryer PE. Hypoglycemia in type 1 diabetes mellitus. Endocrinol M etab Clin North Am. 2010;39(3):64154.

2 Cryer PE. Diverse causes of hypoglycemia-associated autonomic failure in diabetes. N Engl J M ed. 2004;350 (22):2272-9.

3 M oreira T M , G omes E B, Santos J C. Cardiovascular risk factors in young adults with arterial hypertension and/ or diabetes mellitus. Rev G aúcha Enfer m. 2010;31 (4):662-9.

4 M cCrimmon RJ, Sherwin RS. Hypoglycemia in type 1 diabetes. Diabetes. 2011;59(10):2333-9.

5 Curcio R, Lima M H, Torres HC. Elaboration for nursing consultation: focus on the treatment with insulin. Rev G aúcha Enferm. 2009;30(3):552-7. 
6 T he Diabetes Control and Complications Trial Research $\mathrm{G}$ roup. $\mathrm{H}$ ypoglycemia in the D iabetes Control and Complications T rial. Diabetes. 1997;46(2):271-86.

7 Boussageon R, Bejan-Angoulvant T, Saadatian-Elahi $M$, L afont S, Bergeonneau C, Kassai B, et al. Effect of intensive glucoselowering treatment on all cause mortality, cardiovascular death, and microvascular events in type 2 diabetes: meta-analysis of randomised controlled trials. BM J. 2011;343:d4169.

8 Rodacki M, M ilech A, Oliveira JE. C-peptide residual secretion makes differ ence on type 1 diabetes management? Arq Bras E ndocrinol M etabol. 2008;52(2):322-33.

$9 \mathrm{~F}$ ranz M J, Bantle JP, B eebe CA, Brunzell J D, Chiasson $J \mathrm{~L}, \mathrm{G}$ arg A, et al. Evidence-based nutrition principles and recommendations for the treatment and prevention of diabetes and related complications. Diabetes Care. 2002;25(1):148-98.

10 Standards of medical care in diabetes: 2012. Diabetes Care. 2012;35 Suppl 1:S11-63.

11 W iethop BV, Cryer PE. Alanine and terbutaline in treatment of hypoglycemia in ID D M . Diabetes $C$ are. 1993;16(8):1131-6.

12 Gannon M C, N uttall F Q. Effect of a high-protein, low-carbohydrate diet on blood glucose control in people with type 2 diabetes. Diabetes. 2004;53(9): 2375-82.

$13 \mathrm{G}$ annon M C, N uttall F Q, Saeed A, Jordan K, H oover $H$. A $n$ increase in dietary protein improves the blood glucose response in persons with type 2 diabetes. A m J Clin N utr. 2003;78(4):734-41.
$14 \mathrm{M}$ artorella AJ. Iatrogenic hypoglycemia in patients with type 2 diabetes: comparison of insulin analog premixes and human insulin premixes. Postgrad M ed. 2011;123(4):7-16.

15 Alvim RO, Santos PC, Ferreira NE, M ill JG, Krieger JE, Pereira AC. T hioredoxin interacting protein (TXNIP) rs7212 polymorphism is associated with arterial stiffness in the Brazilian general population. J Hum Hypertens. E pub 2011 N ov 24.

16 Ferreira $N E$, Omae $S$, Pereira $A$, Rodrigues $M V_{\text {, }}$ M iyakawa AA, Campos LC, et al. Thioredoxin inter acting protein genetic variation is associated with diabetes and hypertension in the Brazilian general population. A therosclerosis. E pub 2011 Dec 22.

17 Boas LC, Santos CB, Foss-F reitas M C, Pace AE. T he relationship between social support and the social demographic characteristics of people with diabetes mellitus. Rev G aúcha E nferm. 2009;30(3):390-6.

$18 \mathrm{M}$ arshall M C Jr. Overcoming barriers to glycemic control in African A mericans with type-2 diabetes: benefits of insulin therapy. J N atl M ed Assoc. 2007; 99(8):868-75.

19 Santos PC, Alvim RO, Ferreira NE, Sá Cunha R, Krieger JE, M ill JG, et al. Ethnicity and arterial stiffness in Brazil. A m J H ypertens. 2011,24(3):27884.

20 Santos $P C$, Soares RA, N ascimento R M , M achadoCoelho GL, M ill JG, Krieger JE, et al. SLCO1B 1 rs4149056 polymorphism associated with statininduced myopathy is differently distributed according to ethnicity in the Brazilian general population: A merindians as a high risk ethnic group. BM C M ed Genet. 2011;12:136.

Recebido em: 20/ 12/ 2010

A provado em: 01/ 02/ 2012

\author{
Endereço da autora / Dirección del autor / \\ Author's address: \\ Silmara Rodrigues $\mathrm{M}$ achado \\ Rua D ona A dma Jafet, 91, Bela V ista \\ 01308-050, São Paulo, SP \\ E-mail: silmara.machado@hsl.org.br
}

